\title{
XLI. On the saturation constants according to van der waals's equation
}

\author{
John P. Dalton M.A.
}

To cite this article: John P. Dalton M.A. (1907) XLI. On the saturation constants according to van der waals's equation, Philosophical Magazine Series 6, 13:76, 517-524, DOI:

$10.1080 / 14786440709463626$

To link to this article: http://dx.doi.org/10.1080/14786440709463626

册 Published online: 16 Apr 2009.

Submit your article to this journal $[\pi$

Џ Article views: 4

Q View related articles $\square$ 


\section{$\left[\begin{array}{ll}517 & ]\end{array}\right.$}

XLI. On the Saturation Constants according to wan der Waals's Equation. By JoHN P. DatToN, M.A., Carnegie Research Scholar*.

A. Deduction of Constants.

TOR the deduction of the saturation constants $\theta, \pi, \omega_{1}$ and $\omega_{2}$, the following different methods are available and have been used :-

1. M. Pianck's method for the equation of Clausius may be adapted to that of van der Waals. For this purpose an independent variable $\phi$ is chosen, such that

$$
3 \omega_{1}-1=3 r \sin ^{2} \frac{1}{2} \phi, \text { and } 3 \omega_{2}-1=3 r \cos ^{2} \frac{1}{2} \phi .
$$

Since

hence

$$
\frac{8 \theta}{3 \omega_{1}-1}-\frac{3}{\omega_{1}^{2}}=\pi=\frac{\cdot 8 \theta}{3 \omega_{2}-1}-\frac{3}{\omega_{2}{ }^{2}},
$$

$$
r=-\frac{2}{3 \sin ^{2} \phi}\left[\frac{\cos \phi+\sin ^{2} \phi \cdot \log \tan \frac{1}{2} \phi}{\log \tan \frac{1}{2} \phi+\cos \phi}\right] .
$$

Also

$$
\pi=\frac{\delta \omega_{2} \omega_{1}-\omega_{2}-\omega_{1}}{\omega_{1}{ }^{3} \omega_{2}{ }^{2}} .
$$

and

$$
\theta=\frac{\left(\omega_{1}+\omega_{2}\right)\left(3 \omega_{2}-1\right)\left(3 \omega_{1}-1\right)}{8 \omega_{1}{ }^{2} \omega_{2}{ }^{2}} .
$$

When $\phi=90^{\circ}, \omega_{1}=1=\omega_{2}$, hence values of $\phi$ must so be chosen that $90^{\circ}>\phi>0$.

This method was used by A. Stoletow $\ddagger$ who calculated pressures only, and gave the following results :-

\begin{tabular}{|c|c|c|}
\hline$\phi$. & $\theta$ & $\pi$. \\
\hline $80^{\circ}$ & 0.9960 & 0.954 \\
70 & 0.9861 & 0.945 \\
65 & 0.9780 & 0.914 \\
60 & 0.9677 & 0876 \\
55 & 0.9549 & 0.829 \\
50 & 0.9396 & 0.776 \\
\hline
\end{tabular}

* Communicated by Prof. J. P. Kuenen, Ph.D.

† M. Planck, Wied. Ann. Bd. xiii. pp. 535-543 (1881).

$\ddagger$ A. Stoletow, J. de russ. phys. Ges. xiv. p. 167 (1882). 
and also by $A$. Batschinski *, who calculated volumes only:-

\begin{tabular}{|c|c|c|c|}
\hline$\phi$ & $\theta$. & $\omega_{i}$. & $\omega_{2}$ \\
\hline $90^{\circ}$ & 1 & 1 & 1 \\
\hline $40^{\circ}$ & 089894 & 0.6021 & $2 \cdot 3625$ \\
\hline $25^{\circ}$ & $(1.80247$ & 0.5100 & $4 \cdot 1103$ \\
\hline $15^{\circ}$ & $06968^{\circ}$ & 0.4659 & 7.9828 \\
\hline $8^{\circ}$ & 058213 & (1) $4 \cdot 27 \pi$ & 19585 \\
\hline $4^{\circ}$ & 0.48222 & $0.40 \div 8$ & $57 \cdot 30$ \\
\hline $2^{\circ}$ & 0.40712 & 0.3877 & 178.8 \\
\hline $0^{\circ} 15^{\prime}$ & 0.30723 & 0.3709 & 1974 \\
\hline
\end{tabular}

2. The constants may be obtained from the $p v$-isothermals using Maxwell's criterion. This method was employed by $H$. Hilton $\dagger$, who gives the following table :-

\begin{tabular}{|c|c|c|c|}
\hline$\theta$. & $\pi$. & $\omega_{1}$ & $\omega_{2 .}$ \\
\hline 0.937 & 0.778 & 0.360 & 1.84 \\
0.875 & 0.587 & 0.573 & 2.53 \\
0.844 & 0.486 & 0.545 & 3.31 \\
0.750 & 0.281 & 0.495 & 4.83 \\
0.625 & 0.090 & 0.441 & - \\
0.000 & 0048 & 0.18 & - \\
\hline
\end{tabular}

3. They may also be obtained graphically by means of the thermodynamic potential. E. Riecke $\ddagger$ using this method obtained the following:-

\begin{tabular}{|c|c|c|c|}
\hline$\theta$. & $\pi$. & $\omega_{1}$. & $\omega_{20}$ \\
\hline $\begin{array}{l}060 \\
0.65 \\
0.70 \\
0.75 \\
0.80 \\
0.85 \\
0.90 \\
0.95\end{array}$ & $\begin{array}{l}0.109 \\
0.137 \\
0 \cdot 199 \\
0 \cdot 288 \\
0 \cdot 384 \\
0 \cdot 507 \\
0.650 \\
0 \cdot 807\end{array}$ & $\begin{array}{l}0.432 \\
0.448 \\
0 \cdot 466 \\
0.489 \\
0.517 \\
0.553 \\
0.602 \\
0.693\end{array}$ & $\begin{array}{r}16.60 \\
10.90 \\
7.75 \\
5.53 \\
4.14 \\
3.05 \\
2.32 \\
1.78\end{array}$ \\
\hline
\end{tabular}

4. A second graphical method is given by the use of the free energy. This method was adopted by Kamerlingh

* A. Batschinski, Zeits. f. phys. Chemie, xli. p. 743 (1902).

† H. Hilton, Phil. Mág. [6] i. p. 579, and ii. p. 803 (1901).

\pm E. Riecke, Wied. Ann. liii. p. 379 (1894). 
Onnes *, but he does not communicate any results. It was used independently by the author. Isothermal $\psi, v$-curves were constructed by means of the equation

$$
-\frac{1}{p_{\mathrm{K}} r_{\mathrm{K}}} \psi=\frac{8 \theta}{3} \log _{e}(3 \omega-1)+\frac{3}{\omega},
$$

and a double-tangent drawn to each curve. The slope of these tangents gave the values of $\pi$. As it was difficult to read volumes exactly from the curve, values of $\pi$ were substituted in the "reduced" equation of state, and the volumes determined by calculation. The tabulated results were:-

\begin{tabular}{|c|c|c|c|}
\hline$\theta$. & $\pi$ & $\omega_{1}$. & $\omega_{2 \cdot}$ \\
\hline 0.50 & $00: 280$ & 0.4067 & $45 \cdot 62$ \\
\hline $0 \cdot 60$ & 0.0869 & 04326 & $16 \cdot 72$ \\
\hline $0 \cdot 65$ & $0 \cdot 1364$ & 0.4485 & $11 \cdot 1 \overline{6}$ \\
\hline 070 & 0.2007 & 0.4671 & 7.799 \\
\hline $0 \cdot 75$ & 0.2825 & 0.4896 & 5.642 \\
\hline 0.80 & 03840 & 0.5174 & $4 \cdot 162$ \\
\hline 085 & $0 \cdot 5058$ & 0.5532 & $3 \cdot 113$ \\
\hline 0.90 & $0 \cdot 6494$ & $0-6029$ & $2 \cdot 337$ \\
\hline $\begin{array}{l}0.95 \\
1.00\end{array}$ & 00005 & $\begin{array}{l}0.6854 \\
1\end{array}$ & $\begin{array}{l}1.744 \\
1.000\end{array}$ \\
\hline
\end{tabular}

5. Clausius's method + applied by him to the equation

$$
\frac{p}{\mathrm{RT}}=\frac{1}{v-\alpha}-\frac{27(\alpha+\beta)}{8 \theta(v+\beta)^{2}},
$$

may be easily adapted to the present purpose and the table given by him $\ddagger$ (which appears to be very accurate) may be used. He calculates at intervals of $\theta=0.01$ the corresponding values of $\frac{\Pi}{\Pi_{\mathrm{K}}}, \frac{w}{w_{\mathrm{K}}}$, and $\frac{\mathrm{W}}{\mathrm{W}_{\mathrm{K}}}$, where $\mathrm{II}=p / \mathrm{RT}, w=v_{1}-\alpha$, and $\mathrm{W}=v_{2}-\alpha$.

By comparing his equation with that of van der Waals expressed in the form

$$
\frac{p}{\mathrm{R}} \overline{\mathrm{T}}=\frac{1}{v-b}-\frac{a}{\mathrm{RT} v^{2}},
$$

it will be seen that the saturation constants $\pi, \omega_{1}$, and $\omega_{2}$

* Kamerlingh Onnes, Comm. Leid. no. 66, p. 10 (1c00).

+ R. Clausius, Wied. Ann. Bd. xiv. pp. 279-290 and 692-704 (1881).

$\ddagger$ Ibid. pp. 694-5. 
according to van der Waals's equation may be derived from Clausius's table by means of the relations

$$
\text { and } \quad \begin{aligned}
\pi & =\theta \frac{\Pi I}{\Pi}, \\
\omega_{1} & =\frac{2 \frac{v}{w_{\mathrm{K}}}+1}{3}, \\
& \omega_{2}=\frac{2 \frac{\mathrm{W}}{\mathrm{W}_{\mathrm{K}}}+1}{3} .
\end{aligned}
$$

\begin{tabular}{|c|c|c|c|}
\hline$\theta$. & $\pi$ & $\omega_{1}$. & ()$_{2}{ }^{*}$ \\
\hline $0 \cdot 20$ & 0.000001180 & 0.35584 & 448520 \\
\hline 1 & 2646 & $0 \cdot 35714$ & 211560 \\
\hline 2 & $\mathbf{5 4 7 8}$ & 0.35846 & 107120 \\
\hline 3 & $0 \cdot 000010626$ & () 35980 & 57670 \\
\hline 4 & 19536 & $0 \cdot 36116$ & 32760 \\
\hline 5 & 34175 & 0.36254 & 19509 \\
\hline 6 & 57226 & $0 \cdot 363995$ & 12111 \\
\hline 7 & 92232 & $0 \cdot 365: 37$ & $7801 \cdot 7$ \\
\hline 8 & 0.00014367 & 0.36682 & $5193 \cdot 5$ \\
\hline 9 & 21701 & 0.36830 & $3560 \cdot 3$ \\
\hline $0 \cdot 30$ & 31881 & 0.36980 & $2505 \cdot 9$ \\
\hline 1 & 45688 & 035133 & $1806 \cdot 1$ \\
\hline 2 & 64006 & $0 \cdot 37 \cdot 288$ & $1330 \cdot 1$ \\
\hline 3 & 87849 & $0: 37446$ & $998 \cdot 60$ \\
\hline 4 & 0.0011835 & $0 \cdot 37608$ & $763 \cdot 13$ \\
\hline 5 & 15673 & 0.37772 & 59261 \\
\hline 6 & 20435 & 0.37959 & $466 \cdot 98$ \\
\hline 7 & 26265 & $0 \cdot 38109$ & 372.93 \\
\hline 8 & 33318 & $0 \cdot 38 \div 83$ & $301 \cdot 49$ \\
\hline 9 & 417.53 & 0.38460 & $24 i \cdot 49$ \\
\hline $0 \cdot 40$ & 51744 & $0 \cdot 38641$ & $203+63$ \\
\hline 1 & 63464 & 0.38825 & $169 \cdot 83$ \\
\hline 2 & 77095 & 0.39013 & $149 \cdot 80$ \\
\hline 3 & 92833 & $0 \cdot 39 \div 06$ & $121 \cdot 20$ \\
\hline 4 & $0 \cdot 011085$ & 0.39402 & $10: 3 \cdot 58$ \\
\hline 5 & 13134 & 0.30602 & $89 \cdot 147$ \\
\hline 6 & $15+51$ & $0 \cdot 39807$ & $77 \cdot 227$ \\
\hline 7 & 18054 & 0.40017 & $67 \cdot 300$ \\
\hline 8 & 20966 & $0 \cdot 40231$ & 58.969 \\
\hline 9 & 24205 & $0 \cdot 40+51$ & 5] 947 \\
\hline $0 \cdot 50$ & 27789 & 0.40675 & 45.984 \\
\hline 1 & 31738 & $0 \cdot 40905$ & $40 \cdot 891$ \\
\hline 2 & 36073 & $0 \cdot 41141$ & 36.517 \\
\hline 3 & 40812 & $0 \cdot 41383$ & $32 \cdot 741$ \\
\hline 4 & 45975 & 0.41630 & 29.465 \\
\hline 5 & 51581 & $0 \cdot 41884$ & $26 \cdot 609$ \\
\hline 6 & 57646 & 0.42145 & $24 \cdot 109$ \\
\hline 7 & 64188 & 0.42413 & $21 \cdot 913$ \\
\hline $\begin{array}{l}8 \\
9\end{array}$ & $\begin{array}{l}71230 \\
78783\end{array}$ & $\begin{array}{l}0.42687 \\
0 \cdot 4907\end{array}$ & $19 \cdot 973$ \\
\hline & 1000 & $0+2=0,1$ & 10.200 \\
\hline
\end{tabular}

As these constants are frequently used, the fuil table is bere given :- 
according to van der Waals's Equation.

\begin{tabular}{|c|c|c|c|}
\hline$\theta$. & $\pi$ & $\omega_{1}$ & $\omega_{2}$ \\
\hline $0 \cdot 60$ & 0.086868 & 0.43262 & $16 \cdot 730$ \\
\hline 1 & 95508 & 0.43560 & $15 \cdot 366$ \\
\hline 2 & 0.10470 & 0.43869 & $14 \cdot 147$ \\
\hline 3 & 11448 & $0 \cdot 44786$ & $13 \cdot 052$ \\
\hline 4 & 12485 & $0 \cdot 44513$ & $12 \cdot 067$ \\
\hline 5 & 13584 & $0 \cdot 14851$ & $11 \cdot 177$ \\
\hline 6 & 14746 & 0.45201 & $10 \cdot 371$ \\
\hline 7 & 15971 & 0.45561 & $9 \cdot 6387$ \\
\hline 8 & 17262 & 0.45934 & 8.9733 \\
\hline 9 & $186: 0$ & 0.46319 & $8 \cdot 3660$ \\
\hline $0 \cdot 70$ & 20046 & 0.46719 & $7 \cdot 8113$ \\
\hline 1 & 21541 & 0.47135 & $7 \cdot 3027$ \\
\hline 2 & 23108 & 0.47565 & 6.8355 \\
\hline 3 & 24747 & $0 \cdot 48013$ & $6 \cdot 4057$ \\
\hline 4 & 26450 & 0.48478 & 60093 \\
\hline 5 & 28246 & 0.48963 & $5 \cdot 6431$ \\
\hline (3) & 30109 & 0.49469 & $5 \cdot 3041$ \\
\hline 7 & 32048 & 0.49998 & 4.9896 \\
\hline 8 & .54064 & 0.50551 & 4.6978 \\
\hline 9 & 36160 & 0.51131 & $4 \cdot 4259$ \\
\hline $0 \cdot 80$ & 38335 & $0 \cdot 51741$ & $4 \cdot 1725$ \\
\hline 1 & 40593 & 0.52382 & 39356 \\
\hline 2 & 42933 & 0.53058 & $3 \cdot 7141$ \\
\hline 3 & 45353 & $0 \cdot 533773$ & $3 \cdot 5064$ \\
\hline 4 & 47859 & 0.54531 & $3 \cdot 3113$ \\
\hline 5 & 50449 & 0.55336 & $3 \cdot 1276$ \\
\hline 6 & 53126 & 0.56195 & 2.9545 \\
\hline 7 & 55886 & 0.57117 & 27910 \\
\hline 8 & 58736 & 058107 & 26361 \\
\hline 9 & 61674 & 0.59176 & $2 \cdot 4889$ \\
\hline & 64700 & 0.60339 & $\begin{array}{l}20481 \\
0.0151\end{array}$ \\
\hline 2 & 71094 & $\begin{array}{l}0.1012 \\
0.63019\end{array}$ & $2 \cdot 0867$ \\
\hline 3 & 74319 & $0 \cdot 64592$ & $1 \cdot 9633$ \\
\hline 4 & 77708 & 066368 & $1 \cdot 8438$ \\
\hline 5 & 81188 & 0.68412 & $1 \cdot 7271$ \\
\hline 6 & 84762 & 070817 & $1 \cdot 6117$ \\
\hline 7 & 88429 & 0.73746 & $1 \cdot 4957$ \\
\hline 8 & 92192 & 0.77535 & $1 \cdot 3757$ \\
\hline 9 & 96048 & $0 \cdot 83028$ & $1 \cdot 2418$ \\
\hline $1 \cdot 00$ & $1 \cdot 00000$ & 1.00000 & $1 \cdot 0000$ \\
\hline
\end{tabular}

B. Comparison with Empirical Formula.

(1) Vapour-pressure Law.

The values of $\pi$ given above were used to test the wellknown empirical vapour-pressure law of van der Waals, viz.,

$$
\log \pi=f\left(1-\frac{1}{\theta}\right),
$$

where $f$ is a constant. Onnes ${ }^{*}$ found that the pressures he obtained by the "free energy" method obeyed the above law

* Loc. cit. 
witl " fair approximation," and gave $f$ (at low temperatures) a value $=3 \cdot 4$, when $\log \pi$ is expressed in natural logarithms.

In the following, the constant has been calculated for both ordinary and natural logarithms :-

\begin{tabular}{|c|c|c|c|c|}
\hline$\theta^{\circ}$ & $-\log _{10} \pi$ & $-\left(1-\frac{1}{\theta}\right)$ & $f^{\prime}$. & $f$ \\
\hline $\begin{array}{r}0.20 \\
.25 \\
.30 \\
.35 \\
.40 \\
.45 \\
.50 \\
.55 \\
.60 \\
.65 \\
.70 \\
.75 \\
.80 \\
.85 \\
.90 \\
.95 \\
1.00\end{array}$ & $\begin{array}{c}5 \cdot 9281 \\
4 \cdot 4663 \\
3 \cdot 4965 \\
2 \cdot 8049 \\
2 \cdot 2861 \\
1 \cdot 8816 \\
1 \cdot 5561 \\
1 \cdot 2875 \\
1 \cdot 06111 \\
\cdot 86697 \\
\cdot 69797 \\
\cdot 54904 \\
\cdot 41640 \\
\cdot 29715 \\
\cdot 18910 \\
\cdot 0.9051 \\
\cdot 00000\end{array}$ & $\begin{array}{c}4 \cdot 0000 \\
3 \cdot 0000 \\
2 \cdot 3333 \\
1 \cdot 8571 \\
1 \cdot 5000 \\
1 \cdot 2 \cdot 229 \\
1 \cdot 0000 \\
\cdot 81818 \\
\cdot 66667 \\
\cdot 53846 \\
\cdot 42857 \\
\cdot 33333 \\
\cdot 25000 \\
\cdot 17647 \\
\cdot 11111 \\
\cdot 05263 \\
\cdot 00000\end{array}$ & $\begin{array}{r}1 \cdot 4820 \\
1 \cdot 4886 \\
1 \cdot 4985 \\
1 \cdot 5103 \\
1 \cdot 5241 \\
1 \cdot 5395 \\
1 \cdot 5561 \\
1 \cdot 5736 \\
1 \cdot 5917 \\
1 \cdot 6101 \\
1 \cdot 6286 \\
1 \cdot 6471 \\
1 \cdot 6656 \\
1 \cdot 6839 \\
1 \cdot 7019 \\
1 \cdot 7197 \\
\end{array}$ & $\begin{array}{l}3 \cdot 4124 \\
3 \cdot 4280 \\
3 \cdot 4504 \\
3 \cdot 4776 \\
3 \cdot 5094 \\
3 \cdot 5449 \\
3 \cdot 5830 \\
3 \cdot 6 \cdot 234 \\
3 \cdot 6650 \\
3 \cdot 7073 \\
3 \cdot 7500 \\
3 \cdot 7925 \\
3 \cdot 8352 \\
3.8773 \\
3 \cdot 9188 \\
3 \cdot 9597 \\
\\
\end{array}$ \\
\hline
\end{tabular}

Fig. 1 shows graphically the deviation of $f$ from a constant.

(2) The Law of Cailletet and Mathias.

Onnes * found that for the saturation volumes determined by him the law of the diameter held "very well up to quite low temperatures," and A. Batschinski $\uparrow$ obtained by plotting mean density against temperatire a line that did not "differ much from a straight line."

From the table of saturation constants given above, the following figures were derived :-

\begin{tabular}{cl|cc}
$\theta$. & $\frac{1}{\omega_{1}}+\frac{1}{\omega_{2}}$ & $\theta$. & $\frac{1}{\omega_{1}}+\frac{1}{\omega_{2}}$ \\
{$[0 \cdot 00$} & $3 \cdot 0000]$ & $\cdot 60$ & $2 \cdot 3713$ \\
$0 \cdot 20$ & $2 \cdot 8103$ & $\cdot 65$ & $2 \cdot 3191$ \\
$\cdot 25$ & $2 \cdot 7584$ & $\cdot 70$ & $2 \cdot 2685$ \\
.30 & $2 \cdot 7046$ & .75 & $2 \cdot 2196$ \\
.35 & $2 \cdot 6492$ & .80 & $2 \cdot 1724$ \\
.40 & $2 \cdot 5928$ & $\cdot 85$ & $2 \cdot 1268$ \\
$\cdot 45$ & $2 \cdot 5363$ & .90 & $2 \cdot 0831$ \\
.50 & $2 \cdot 4802$ & .95 & $2 \cdot 0407$ \\
.55 & $2 \cdot 42.51$ & $1 \cdot 00$ & $2 \cdot 0000$
\end{tabular}

* Loc. cit.

$\dagger$ Loc. cit. 
according to van der Waals's Equation.

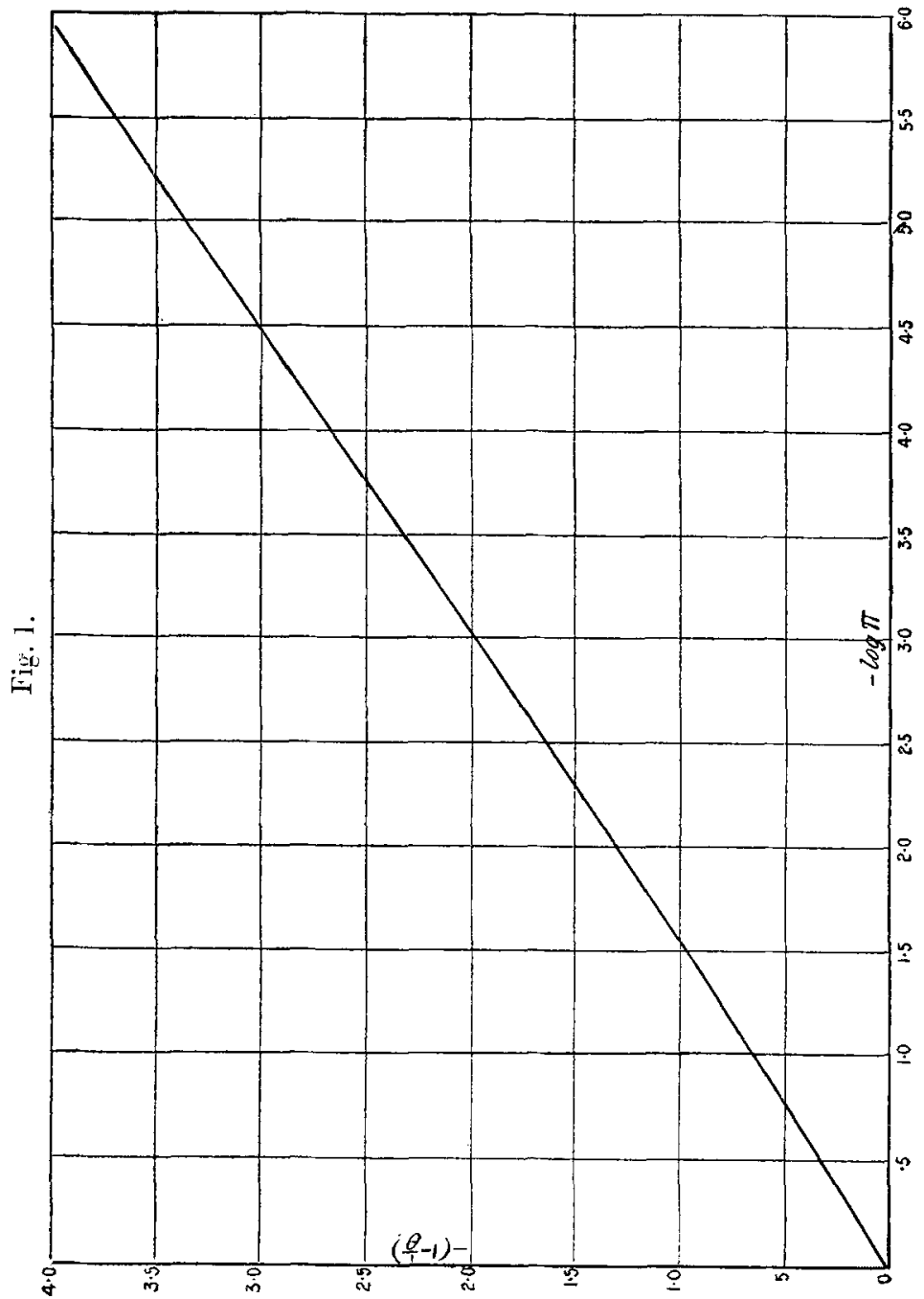

It is known * that the equation

$$
\frac{1}{\omega_{1}}+\frac{1}{\omega_{2}}=a+b \theta
$$

is not an accurate expression of the results of observation, but the curvature shown by plotting the foregoing numbers * S. Young, Phil. Mag. [5] 1. pp. 291-305 (1900). 
524 Saturation Constants and van der Waals's Equation.

(fig. 2) is more marked than that given by experimental results.

Fig. 2.

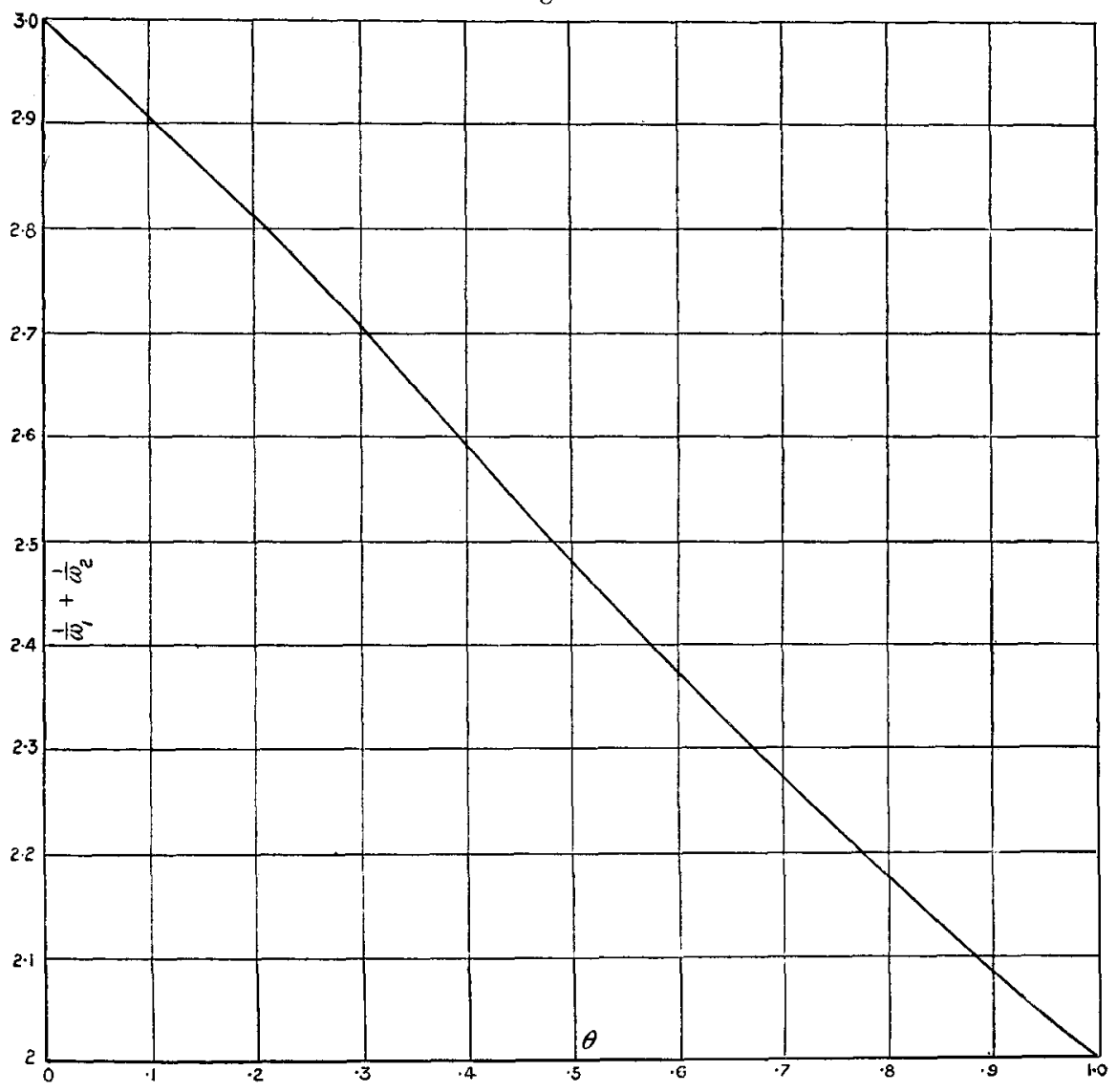

S. Young (loc. cit.) finds as a more correct formula

$$
\mathrm{D}_{t}=\mathrm{D}_{0}+\alpha t+\beta t^{2} \text {. }
$$

For all substances $\alpha$ is negative; $\beta$ is zero only when the ratio of the actual to the theoretical density at the critical point has the normal value $3 \cdot 77$. When this ratio $<3.77, \beta$ is positive and the curve is convex to the temperature-axis. This is quite in accordance with fig. 2 , where the ratio has the value $2 \cdot 67$.

University College, Dundee,

February, 1907. 